\title{
Preoperative percutaneous coronary intervention in patients undergoing open thoracoabdominal and descending thoracic aneurysm repair
}

\author{
Leonard N. Girardi, MD, Yury Rabotnikov, MD, and Dimitrios V. Avgerinos, MD
}

\begin{abstract}
Objective: Current guidelines have recommended against coronary revascularization before noncardiac surgery in patients with asymptomatic coronary artery disease. However, myocardial infarction after thoracic aneurysm (TA) repair dramatically increases the morbidity and mortality. Revascularization with coronary artery bypass grafting before TA repair minimizes the incidence of perioperative ischemia. However, the recovery can be prolonged, and a percentage of patients will either never return for aneurysm repair or will develop a rupture during convalescence. Percutaneous coronary intervention (PCI) before TA repair might be preferable. Previous studies examining PCI before major vascular surgery included few patients with TAs. We examined the outcomes of patients undergoing PCI before TA repair.
\end{abstract}

Methods: From 1997 to 2012, 592 patients underwent TA repair. Patients presenting for elective repair underwent cardiac catheterization before surgery. Those with significant single- or double-vessel coronary artery disease underwent PCI. The perioperative outcomes were examined and compared with those of patients undergoing TA repair without revascularization.

Results: A total of 44 patients $(7.4 \%)$ underwent PCI with bare metal stents before surgery. No PCI-related complications occurred. Dual antiplatelet therapy was administered for 4 to 6 weeks. No instances of aneurysm rupture occurred in the interval between PCI and surgery. The incidence of stent thrombosis, myocardial infarction, and mortality for those undergoing PCI was 0 . No bleeding complications occurred.

Conclusions: PCI is safe and efficacious in patients undergoing TA repair. Aneurysm rupture did not occur in the interval before surgery. Antiplatelet therapy did not increase the risk of bleeding complications. Stent thrombosis was not seen. We recommend PCI those with significant single- or double-vessel coronary artery disease before elective TA repair. (J Thorac Cardiovasc Surg 2014;147:163-8)

According to the current guidelines from the American College of Cardiology and the American Heart Association (ACC/AHA), coronary revascularization before elective noncardiac surgery is not recommended. ${ }^{1}$ The results of randomized, controlled trials have failed to demonstrate a reduction in the incidence of perioperative myocardial infarction (MI) or an improvement in long-term survival when percutaneous coronary intervention (PCI) was performed before major vascular surgery, including abdominal aortic aneurysm (AAA) repair. ${ }^{2,3}$ Despite these recommendations, however, controversy has persisted regarding the benefits of preoperative myocardial

From the Department of Cardiothoracic Surgery, Weill Cornell Medical College, New York, NY

Disclosures: Authors have nothing to disclose with regard to commercial support.

Read at the 93rd Annual Meeting of The American Association for Thoracic Surgery, Minneapolis, Minnesota, May 4-8, 2013.

Received for publication May 10, 2013; revisions received Aug 20, 2013; accepted for publication Sept 4, 2013; available ahead of print Oct 30, 2013.

Address for reprints: Leonard N. Girardi, MD, Department of Cardiothoracic Surgery, Weill Cornell Medical College, 525 E 68th St, Suite M404, New York, NY 10065 (E-mail: lngirard@med.cornell.edu).

$0022-5223 / \$ 36.00$

Copyright (c) 2014 by The American Association for Thoracic Surgery

http://dx.doi.org/10.1016/j.jtcvs.2013.09.008 revascularization in a higher risk cohort of patients undergoing major vascular surgery., ${ }^{2,4}$ In particular, those undergoing aortic reconstruction are a particularly high-risk group with a significantly increased incidence of lethal perioperative MI. ${ }^{4}$ When revascularization precedes surgery in these higher risk cohorts, patients derive not only a reduction in their perioperative mortality, but also improvement in their long-term survival. ${ }^{5}$

Patients undergoing either descending thoracic aneurysm (DTA) or thoracoabdominal aortic aneurysm (TAAA) repair constitute an even higher risk cohort requiring major vascular surgery. Proximal aortic crossclamping, blood loss, and rapid volume shifts create greater degrees of myocardial work, increasing the risk of significant myocardial ischemia. Such dramatic shifts in the hemodynamics increase intracoronary wall shear stress and can precipitate endothelial damage, vulnerable plaque rupture, and acute MI. ${ }^{6,7}$ In patients undergoing emergent aneurysm repair for thoracic aortic rupture, the increased risk of perioperative ischemia was evident in the $100 \%$ mortality seen in patients experiencing a MI either during or after repair of their ruptured aneurysm. ${ }^{8}$ Before the present experience, we had adopted a strategy of cardiac catheterization before elective TA repair. Those with 


\section{Abbreviations and Acronyms \\ AAA $=$ abdominal aortic aneurysm \\ ACC/AHA $=$ American College of Cardiology and the American Heart Association

$\begin{array}{ll}\text { CABG } & =\text { coronary artery bypass grafting } \\ \text { CAD } & =\text { coronary artery disease } \\ \text { DTA } & =\text { descending thoracic aneurysm } \\ \text { MI } & =\text { myocardial infarction } \\ \text { PCI } & =\text { percutaneous coronary intervention } \\ \text { TAAA } & =\text { thoracoabdominal aortic aneurysm } \\ \text { TA } & =\text { thoracic aneurysm }\end{array}$

significant coronary artery disease (CAD) underwent preoperative myocardial revascularization with either coronary artery bypass grafting (CABG) or PCI. Although we were content with our results with these patients, a report of catastrophic stent thrombosis in patients undergoing PCI before thoracoabdominal aneurysm repair stimulated us to examine our practice in more detail. ${ }^{9}$ The possibility of aneurysm rupture during the post-PCI period was also of concern. We sought to examine the safety and efficacy of PCI before elective, open repair of aneurysms of the thoracoabdominal or descending thoracic aorta.

\section{METHODS \\ Patients}

A retrospective review of the department of cardiothoracic surgery aortic surgery database was conducted to identify patients who had undergone either DTA or TAAA repair from July 1997 to July 2012. The institutional review board approved the study and waived the need for individual patient consent.

During the study period, a total of 592 cases were performed: 407 TAAA and 185 DTA. Of the 592 cases, 87 patients presented with aortic rupture and an additional 33 presented with acute aortic syndromes that required urgent intervention negating a full cardiac evaluation before surgery. These 120 patients were excluded from the present analysis, leaving 472 patients with a cardiac evaluation before surgery available.

\section{Preoperative Cardiac Evaluation}

Of the 472 patients, $28(5.9 \%)$ were $<40$ years old with a known connective tissue disorder and normal or mildly reduced left ventricular function. These patients underwent nuclear stress imaging. No evidence was found of ischemia on any of these examinations, and cardiac catheterization was not performed. The remaining 444 patients underwent cardiac catheterization before TA repair. Patients identified with severe left main CAD $(n=9)$ and those with significant triple-vessel CAD $(n=22)$ underwent CABG $(\mathrm{n}=31,6.6 \%)$ before aneurysm repair. An additional 44 patients $(9.3 \%)$ were identified with either significant ( $>70 \%$ stenosis) single- or doublevessel disease and were referred for PCI either during the initial angiogram or the following day. The demographics and cardiac risk factors of the patients undergoing PCI are listed in Table 1. Patients undergoing PCI had a significantly greater mean aneurysm diameter than those not undergoing preoperative revascularization $(8.3 \mathrm{~cm}$ vs $6.9 \mathrm{~cm} ; P<.001)$.

\section{Percutaneous Coronary Intervention}

All patients undergoing PCI were treated with bare metal stents. Their angina status before PCI are listed in Table 2. None of the patients presented with acute MI. Most $(86 \%)$ required only single-vessel intervention; $14 \%$ needed double-vessel PCI. Before stent insertion, the patients were given a loading dose of $300 \mathrm{mg}$ of clopidogrel. Double antiplatelet therapy (75 $\mathrm{mg}$ clopidogrel and $81 \mathrm{mg}$ aspirin) was continued for a mean of 4.6 weeks (range, 3.3-8) in this study group. Clopidogrel was discontinued 1 week before the patient's planned aortic reconstruction. Aspirin was continued until the day before surgery and was resumed after spinal drain removal.

\section{Anesthesia, Surgical Procedures, and Postoperative Care}

General anesthesia was induced with intravenous midazolam hydrochloride and sodium pentothal or propofol, followed by fentanyl citrate boluses. Paralysis was maintained using vecuronium bromide. All patients received invasive hemodynamic monitoring with an arterial line and right heart catheter. Single lung ventilation was achieved with a bronchial blocker. A spinal drain was inserted between the fourth and fifth lumber vertebrae, and the intrathecal pressure was maintained at $<12 \mathrm{~mm} \mathrm{Hg}$ throughout the procedure and for the first 72 hours postoperatively (maximum, $25 \mathrm{~mL}$ of spinal fluid drained per hour).

A fifth intercostal space thoracotomy or thoracoabdominal incision was used in all patients. For those undergoing surgery with either a clamp and sew technique or the assistance of left heart bypass, $100 \mathrm{IU} / \mathrm{kg}$ of heparin was administered, with subsequent boluses given to maintain an activated clotting time $>300$ seconds. The core temperature was allowed to decrease to $33^{\circ} \mathrm{C}$ before crossclamping. Left heart bypass was used for those patients with compromised left ventricular function (ejection fraction $>40 \%$ ), all extent II aneurysms, and those with chronic dissection. The flow was maintained at $3 \mathrm{~L} / \mathrm{min}$. Aggressive red blood cell salvage was used to return lost blood volume. When proximal aortic control was unattainable, circulatory arrest was used. For circulatory arrest, $400 \mathrm{IU} / \mathrm{kg}$ of heparin was given, femoral vein to femoral artery cardiopulmonary bypass was initiated, and the patients were cooled to a core temperature of $18^{\circ} \mathrm{C}$. Rewarming was achieved, maintaining a blood temperature to core temperature gradient of $10^{\circ} \mathrm{C}$.

Aortic reconstruction was performed with Hemashield Dacron grafts (Macquet Corp, Oakland, NJ). After completion of the procedure, protamine sulfate was administered to reverse the anticoagulation, and blood products were administered as necessary to reverse the coagulopathy. In the intensive care unit, the mean arterial pressure was maintained at $>85 \mathrm{~mm} \mathrm{Hg}$ for the first 72 hours postoperatively. Inotropes were added as indicated. The spinal drain was removed on postoperative day 3 . All patients were assessed for myocardial ischemia with serial electrocardiographic monitoring. Those who had undergone previous PCI or CABG and those with known moderate CAD were also evaluated with serial serum cardiac enzyme analysis. A new MI was defined as new Q waves or ST-segment elevation on the electrocardiogram or by a troponin I level $>0.04 \mathrm{mg} / \mathrm{mL}$.

\section{Statistical Analysis}

All the data collected from 1997 to 2012 were stored using Microsoft Access (2010) software (Microsoft, Redmond, Wash). Processing and analysis of the data were performed using IBM SPSS Statistics, version 20 (IBM, Armonk, NY). The chi-square test and Student's $t$ test were used. $P \leq .05$ was considered statistically significant.

\section{RESULTS}

A total of 44 patients underwent PCI before repair of their TA. The stented vessels are listed in Table 2. No myocardial, cerebrovascular, or access complications occurred related to either diagnostic angiography or PCI. No postintervention MIs occurred, and all patients 
TABLE 1. Preoperative patient demographics

\begin{tabular}{lc}
\hline \multicolumn{1}{c}{ Variable } & Value \\
\hline Total & 44 \\
Female gender & $32(73)$ \\
Age (y) & \\
$\quad$ Mean & 72.6 \\
$\quad$ Range & $38-92$ \\
Ejection fraction (\%) & \\
Mean & 47 \\
Range & $20-60$ \\
Mean aneurysm diameter (cm) & $8.3 \pm 1.3$ \\
NYHA class & \\
I & $36(82)$ \\
II & $4(9)$ \\
III & $1(2)$ \\
IV & $3(7)$ \\
CAD risk factors & \\
Smoking & $28(63)$ \\
Hyperlipidemia & $11(25)$ \\
Diabetes mellitus & $6(14)$ \\
Hypertension & $44(100)$ \\
Family history of CAD & $5(11)$ \\
\hline Data presented as n (\%), unless otherwise noted. NYHA, New & York Heart \\
Association; CAD, coronary artery disease. &
\end{tabular}

remained asymptomatic until their aneurysm repair. All 44 patients returned within 8 weeks for their aneurysm repair. No patient experienced aneurysm rupture in the interval between PCI and surgery.

An additional 31 patients underwent CABG before their TAAA repair. No perioperative strokes or mortalities occurred related to that procedure. No aneurysm rupture occurred in the interim between CABG and aneurysm repair, although the mean interval between the 2 procedures was 3 months. After TAAA repair, no MIs occurred and no graft occlusion was evident in the perioperative period.

The operative procedures, techniques, and postoperative complications of the study cohort are listed in Table 3. Of the 31 patients undergoing TAAA repair, $21(68 \%)$ had Crawford extent I and II aneurysms. Of the 13 patients undergoing DTA repair, $8(61 \%)$ required replacement of

TABLE 2. Anginal status and PCI target vessels

\begin{tabular}{lc}
\hline \multicolumn{1}{c}{ Variable } & Patients (n) \\
\hline Anginal status at presentation & \\
Asymptomatic & $36(81)$ \\
Stable angina & $4(9)$ \\
Unstable angina & $4(9)$ \\
Acute MI & $0(0)$ \\
Coronary artery stented & \\
LAD & $12(27)$ \\
Circumflex & $15(34)$ \\
RCA & $11(25)$ \\
RCA and circumflex & $6(14)$ \\
\hline Data in parentheses are percentages. $P C I$, Percutaneous coronary intervention; \\
$M I$, myocardial infarction; $L A D$, left anterior descending; $R C A$, right coronary artery.
\end{tabular}

TABLE 3. Aneurysm type, operative technique, and in-hospital morbidity and mortality

\begin{tabular}{lc}
\hline \multicolumn{1}{c}{ Variable } & Patients (n) \\
\hline Aneurysm type & \\
Extent I TAAA & $15(34)$ \\
Extent II TAAA & $6(14)$ \\
Extent III TAAA & $8(18)$ \\
Extent IV TAAA & $2(4)$ \\
DTA & $13(30)$ \\
Operative technique & \\
Clamp and sew & $34(77)$ \\
Left heart bypass & $9(20)$ \\
Circulatory arrest & $1(3)$ \\
Morbidity and mortality & \\
MI & 0 \\
CVA & $1(2)$ \\
SCI & $1(2)$ \\
Return to OR for bleeding & 0 \\
Acute renal failure or HD & $2(4.5)$ \\
Tracheostomy & $2(5)$ \\
Mortality & 0 \\
\hline Data in parentheses are percentages. TAAA, Thoracoabdominal & aneurysm; \\
$D T A$, descending thoracic aneurysm; $M I$, myocardial infarction; $C V A$, cerebro- \\
vascular accident; $S C I$, spinal cord injury; $O R$, operating room; $H D$, hemodialysis.
\end{tabular}

their entire descending aorta. Of the 44 patients, 34 $(77 \%)$ had their aneurysms repaired using a clamp and sew technique. The remainder underwent repair with the assistance of left heart bypass $(n=9,20 \%)$ or circulatory arrest $(\mathrm{n}=1,3 \%)$.

No perioperative MIs or instances of stent thrombosis occurred. No patient required a return to the operating room for postoperative hemorrhage. The 1 patient experiencing a cerebrovascular accident $(2.3 \%)$ recovered completely without deficit before discharge. The 1 patient with paraplegia after surgery $(2.3 \%)$ had had an extent IV aneurysm repaired with a clamp and sew technique and a crossclamp time of 30 minutes. He has remained paralyzed. Two patients $(4.5 \%)$ required tracheostomy after prolonged intubation. Two patients $(4.5 \%)$ requiring renal replacement therapy after aneurysm repair recovered their renal function before discharge. No 30-day or in-hospital mortality occurred among the 44 patients undergoing PCI before aneurysm repair.

Univariate analysis comparing elective patients undergoing preoperative PCI (group 1) and those undergoing either preoperative $\mathrm{CABG}$ (group 2) or no revascularization (group 3 ) failed to demonstrate a significant difference among the groups with respect to mortality $(P<.33)$, MI $(P<1.0)$, or spinal cord injury $(P<.7)$. Those undergoing PCI did have a significantly greater incidence of cerebrovascular accident compared with group 3 (no revascularization; $P<.027$ ).

\section{DISCUSSION}

The most recent ACC/AHA guidelines for perioperative cardiovascular evaluation and care for the noncardiac 
surgery patient were published in $2007 .{ }^{1}$ Based on extensive review of the published data, these recommendations guide the need for preoperative cardiac evaluation and revascularization according to the patient's symptoms and/or the presence of threatening obstructive CAD. Specific references are made to the patient undergoing major vascular surgery, including those requiring AAA repair, because $\geq 50 \%$ of these patients have been shown to have significant CAD. ${ }^{10}$ In symptomatic patients with known left main or triple vessel disease, revascularization before surgery has been strongly recommended. Similar class I, level of evidence A recommendations have been made for patients meeting the following criteria: symptoms with 2-vessel disease involving the proximal left anterior descending artery, symptoms with an ejection fraction $<50 \%$, or ischemia on stress testing. Patients with angina who require elective surgery within the next 12 months, such as the $19 \%$ of patients in our study, should undergo PCI, if possible, followed by 4 to 6 weeks of dual antiplatelet therapy before proceeding. However, $81 \%$ of the patients in our series were asymptomatic with respect to their CAD. The current guidelines have recommended against preoperative invasive testing and revascularization. These class III, level of evidence B recommendations suggest that this cohort of patients will not only fail to benefit from preoperative revascularization, but also might actually be harmed during either the evaluation or treatment of CAD before surgery.

The AHA/ACC guidelines were developed after randomized, prospective trials failed to show a benefit for coronary revascularization in patients needing major vascular surgery. In 2004, the Coronary Artery Revascularization Prophylaxis trial reported on 510 patients in need of vascular surgery, one half of whom were randomized to coronary revascularization before their procedure. ${ }^{3}$ Despite preoperative $\mathrm{PCI}$ or $\mathrm{CABG}, 12 \%$ of these patients experienced a MI after surgery and $14 \%$ without revascularization had a similar outcome. At a median follow-up of 2.7 years, no significant difference was found in survival $(22 \%$ vs $23 \%$ ). However, this highly selected group of patients excluded those with left main disease or a reduced ejection fraction. In addition, only one third of the patients had undergone AAA repair, a population that has been shown to have a significantly greater risk than those undergoing infrainguinal vascular reconstruction. ${ }^{4}$ When analyzing those higher risk patients undergoing AAA repair, a significantly greater incidence of MI after surgery $(19 \%$ vs $7.5 \%, P=.01$ ) was found. The short- and long-term mortality for the patients with an AAA was not reported.

A second randomized study, the Decrease- $V$ pilot study, also randomly assigned preoperative revascularization to 101 patients scheduled for major vascular surgery. ${ }^{2}$ Again, fewer than one third of the patients were in the high-risk category undergoing AAA repair. Despite a trend toward a greater composite endpoint of all-cause mortality or MI at 30 days $(43 \%$ vs $33 \%, P=.3)$, the investigators concluded that preoperative revascularization was not associated with improved outcomes. Despite the acknowledgement that the study had been severely underpowered, these data, and the data from the Coronary Artery Revascularization Prophylaxis, were highly influential in the development of the AHA/ACC guidelines.

Examining a higher risk cohort of patients, Monaco and colleagues $^{5}$ reported on 208 consecutive moderate- to high-risk patients scheduled for elective major vascular surgery. The patients were randomized to either selective coronary angiography because of positive noninvasive testing findings or routine systematic preoperative coronary angiography. Of the 103 patients in the selective group, 46 subsequently underwent cardiac catheterization, with most requiring either $\mathrm{PCI}$ or $\mathrm{CABG}$ before their vascular surgery. No perioperative MIs or mortalities occurred in that group. Of the remaining 57 patients not undergoing catheterization because of negative stress imaging findings, the mortality was $6.5 \%$, with all deaths from cardiac causes. In those undergoing mandatory catheterization before high-risk vascular surgery, $60 \%$ required revascularization. Again, no perioperative MIs or mortalities occurred in that group. Furthermore, in the group undergoing mandatory catheterization, a 53.6\% relative risk reduction from any cardiac-related events out to 8 years was found. These same patients also benefitted from a $16.7 \%$ absolute reduction in all-cause mortality out to 4 years compared with those patients undergoing selective angiography only.

Patients undergoing DTA and TAAA repair are clearly a higher risk cohort than even those undergoing AAA repair. Proximal aortic crossclamping generates rapid increases in the left ventricular systolic and diastolic dimensions, myocardial wall stress, mean arterial pressure, and heart rate. ${ }^{11}$ Such dramatic hemodynamic changes can precipitate acute MI by increasing intracoronary wall shear stress, endothelial damage, and vulnerable plaque rupture. ${ }^{6,7}$ The importance of maximizing the perioperative cardiac function, including the prevention of MI, is highlighted when examining the role of perioperative cardiac complications on outcomes in patients undergoing major thoracic aortic reconstruction. In a contemporary report of 509 patients undergoing elective TAAA repair, Wong and colleagues ${ }^{12}$ reported that $26.7 \%$ of their patients had a cardiac complication after surgery. These patients had a $25.7 \%$ mortality rate compared with $1.3 \%$ mortality in those who did not. ${ }^{12}$ In patients undergoing emergent repair of ruptured DTA or TAAAs, the influence of perioperative ischemia will be even more pronounced. Nearly $60 \%$ of the mortalities in our experience were secondary to an acute MI, and all 4 patients experiencing an MI died either in the operating room or within 48 hours in the intensive care unit. ${ }^{8}$ 
A reduction in the incidence of MI will not only reduce the mortality but has also been shown to reduce the risk of spinal cord dysfunction after thoracoabdominal aneurysm repair. $^{13}$

Two additional concerns for patients undergoing PCI before major vascular surgery are the potential for stent thrombosis in the perioperative period and aneurysm rupture in the interim between revascularization and surgery. An earlier report by Kaluza and colleagues ${ }^{9}$ detailed catastrophic stent thrombosis in patients undergoing vascular surgery, including TAAA repair, within 2 weeks of bare metal stent placement. Of 40 patients in their series, 7 sustained a perioperative MI secondary to stent thrombosis. Of the 7 MIs, $6(86 \%)$ were fatal, with 1 patient surviving after emergent angioplasty. A more contemporary and larger experience with this same cohort of patients showed similarly fatal outcomes, with $75 \%$ mortality in those experiencing stent thrombosis after noncardiac surgery. ${ }^{14}$ However, a more detailed analysis of these data showed that none of the events occurred when the patients had been treated for 6 weeks with dual antiplatelet therapy before proceeding to surgery. Stent placement results in denudation of the coronary artery endothelium. Re-endothelialization occurs within 8 weeks of PCI and even 2 weeks of therapy with a thienopyridine, such as clopidogrel, substantially reduces the risk of acute stent thrombosis. ${ }^{15,16}$ The safety of percutaneous coronary revascularization is epitomized in our series. None of the patients experienced a complication related to angiography or stent placement. After a mean delay of 4.6 weeks after PCI, clopidogrel was stopped and aspirin therapy was continued throughout the perioperative period. Not a single incident of perioperative stent thrombosis or MI was seen in the patients receiving this form of therapy before DTA or TAAA repair. Just as important, no instances of aneurysm rupture occurred while waiting for stent endothelialization.

The results of the present study support an aggressive approach to treating CAD before DTA or TAAA repair. Preoperative coronary angiography and PCI with bare metal stents is safe. Delaying surgery for 4 to 6 weeks will allow the stents to endothelialize and will not increase the risk of aneurysm rupture. Despite the hypercoagulable state often seen in the period after aneurysm repair, ${ }^{17} \mathrm{PCI}$ protected patients against MI, a highly mortal event after elective and emergent TA repair. In addition, dual antiplatelet therapy did not result in an increase in perioperative bleeding complications.

The limitations of the present study included the small sample size and the retrospective nature of our investigation. In addition, no contemporary comparative study cohort was available to evaluate cardiac complications and their influence on outcomes in patients undergoing high-risk, open TA repair without treatment of known, significant CAD.

\section{CONCLUSIONS}

Patients undergoing elective TA repair have a significant incidence of silent CAD. Perioperative MI substantially increases the morbidity and mortality of these operations. The incidence of these events can be dramatically reduced in patients with single- or double-vessel CAD by performing PCI before aneurysm repair. Although these data were from a small cohort of nonrandomized patients, consideration should be given to modifying the ACC/AHA guidelines to reflect the safety and efficacy of PCI before elective TA repair.

\section{References}

1. Fleisher LA, Beckman JA, Brown KA, Calkins H, Chaikof EL, Fleischmann KE, et al. 2009 ACCF/AHA focused update on perioperative beta blockade incorporated into the ACC/AHA 2007 guidelines on perioperative cardiovascular evaluation and care for noncardiac surgery. J Am Coll Cardiol. 2009:54:e13-118.

2. Poldermans D, Schouten O, Didakovic R, Bax JJ, Thomson IR, Howks SE, et al. A clinical randomized trial to evaluate the safety of a noninvasive approach in high-risk patients undergoing major vascular surgery. J Am Coll Cardiol. 2007;49:1763-9.

3. McFalls EO, Ward HB, Moritz TE, Goldman S, Krupski WC, Littooy F, et al. Coronary-artery revascularization before elective major vascular surgery N Engl J Med. 2004;351:2795-804

4. Fleisher LA, Eagle KA, Shaffer T, Anderson GF. Perioperative and long-term mortality rates after major vascular surgery: the relationship to preoperative testing in the Medicare population. Anesth Analg. 1999;89:849-55.

5. Monaco M, Stassano P, Di Tommaso L, Pepino P, Giordano A, Pinna GB, et al Systemic strategy of prophylactic coronary angiography improves long-term outcome after major vascular surgery in medium- to high-risk patients. J Am Coll Cardiol. 2009;54:989-96

6. Gertz SD, Roberts WC. Hemodynamic shear force in rupture of coronary arterial atherosclerotic plaques. Am J Cardiol. 1990;66:1368-72.

7. Fukumoto Y, Hiro T, Fujii T, Hashimoto G, Fujimura T, Yamada J, et al Localized elevation of shear stress is related to coronary plaque rupture. J Am Coll Cardiol. 2008;51:645-50.

8. Girardi LN, Krieger KH, Altorki NK, Mack CA, Lee LY, Isom OW. Ruptured descending and thoracoabdominal aortic aneurysms. Ann Thorac Surg. 2002; 74:1066-70.

9. Kaluza GL, Joseph J, Lee JR, Raizner ME, Raizner AE. Catastrophic outcomes of noncardiac surgery soon after coronary stenting. J Am Coll Cardiol. 2000;35 1288-94.

10. Hertzer NR, Young IR, Kramer JR, Phillips DF, deWolfe VG, Ruschhaupt WF III, et al. Routine coronary angiography prior to elective aortic reconstruction: results of selective myocardial revascularization in patients with peripheral vascular disease. Arch Surg. 1979;114:1336-44.

11. Aakhus S, Aadahl P, Stromholm T, Myhre HO. Increased left ventricula contractility during cross-clamping of the descending thoracic aorta. J Cardiothorac Vasc Anesth. 1995;9:497-502.

12. Wong DR, Parenti JL, Green SY, Chowdhary V, Liao JM, Zarda S, et al Open repair of thoracoabdominal aortic aneurysm in the modern surgical era: contemporary outcomes in 509 patients. J Am Coll Surg. 2011;212:569-81.

13. Acher CW, Wynn MM, Hoch JR, Kranner PW. Cardiac function is a risk factor for paralysis in thoracoabdominal aortic replacement. J Vasc Surg. 1998;27: 821-8.

14. Wilson SH, Fasseas P, Orford JL, Lennon RJ, Horlocker T, Charnoff NE Clinical outcome of patients undergoing non-cardiac surgery in the two months following coronary stenting. J Am Coll Cardiol. 2003;42:234-40.

15. Ueda Y, Nanto S, Komamura K, Kodama K. Neointimal coverage of stents in human coronary arteries observed by angioscopy. J Am Coll Cardiol. 1994;23: 341-6. 
16. Berger PB, Bell MR, Grill DE, Melby S, Holmes DR Jr. Safety and efficacy of ticlopidine for only two weeks after successful intracoronary stent placement. Circulation. 1999;99:248-53.

17. Bradbury A, Adam D, Garrioch M, Brittenden J, Gillies T, Ruckley CV. Changes in platelet count, coagulation and fibrinogen associated with elective repair of asymptomatic abdominal aortic aneurysm and aortic reconstruction for occlusive disease. Eur J Vasc Endovasc Surg. 1997;13:375-80.

\section{Discussion}

Dr Anthony L. Estrera (Houston, Tex). Very nice and clear presentation, Len.

The ACC and AHA have provided us with guidelines for invasive testing for $\mathrm{CAD}$ before noncardiac major vascular surgery based primarily on clinical status; that is, the presence of symptoms and the functional status, as represented by metabolic equivalents or METS, and have recommended coronary artery intervention for patients with a reduced ejection fraction and multivessel disease and those with left main disease.

This was based on level 1 data from randomized trials, as you had mentioned, on major vascular surgery, albeit, few or no patients were included with TAAAs.

Dr Girardi has adopted a policy to perform cardiac catheterization for most of these patients before TAAA repair, and that 1 - or 2-vessel disease, if discovered, should receive intervention with PCI using a bare metal stent.

You demonstrated excellent results in this group of 44 patients, with no postoperative MI, no stent thrombosis, no bleeding, and no death. However, I am still concerned about opposing the guidelines of the AHA and ACC.

I have 2 questions. First, as suggested in your protocol, most patients underwent cardiac catheterization before TAAA repair. With concern for postoperative renal failure, although you had a low percentage of it, what was the interval between heart catheterization and repair, and what is your current policy regarding this?

Dr Girardi. In the patients who are coming in for elective repair, just as for any other aneurysm repair, we will perform catheterization the day before. However, we ask for only a coronary angiogram and a right heart catheterization. We do not perform a formal aortogram or a V-gram. We try to keep the contrast to a minimum, and we have not found that to be a problem contributing to renal failure postoperatively.

Thus, in most of the patients, we will perform catheterization the day before just as if they were going to undergo valve surgery or coronary bypass.

Dr Estrera. Second question. In a report from our group, we suggested that patients with normal functional capacity and a normal left ventricular ejection fraction did not require additional workup, just as has been suggested by the ACC and AHA. In your study, $82 \%$ of the study cohort were asymptomatic and would not have normally undergone an invasive evaluation.
Without a true control group, how do you really know that these patients would not have done well without PCI, and should this have tempered your conclusions?

Dr Girardi. Well, certainly I think 1 of the reasons that we started studying this was exactly as you had stated, our concern for actually going against the recommendations of the AHA/ACC guidelines.

Now, the IIIb recommendations are pretty strong, especially when they start talking about the potential harm associated with the procedure or with PCI itself. We wanted to ensure that, at the very minimum, we were not hurting anybody, and I think we have shown that we have not. We have not hurt these patients with the procedure itself. We also have not hurt them with complications from the stenting.

The only point that we can use to state that we are really providing patients with a benefit is when you consider the historical controls of TA repair, and the incidence of MI, although not crazy, is still pretty high, $5 \%$ or $6 \%$. Also, if the patients develop these complications, it has been a strong predictor for a bad outcome. For those patients who we took to the operating room emergently who did experience a MI, the MIs were almost all uniformly fatal.

The stresses of TA repair as far as the hemodynamics, as far as the cardiac work and the amount of ischemia generated is far different than what we will see in patients undergoing peripheral vascular surgery, from whom most of these data were generated.

Thus, we think it is justified. Certainly, if we can do better at protecting them from a potentially fatal complication such as MI, we think it is worthwhile.

Dr Estrera. Nice job, Len.

Dr Marc R. Moon (St Louis, Mo). It was not clear to me, but did you have any functional analysis that could have, potentially-for example, stress tests - that could have ruled out most of the patients so that you did not have to perform catheterization on everybody?

Dr Girardi. We did not perform stress tests for all the patients. We were very aggressive with catheterization.

One of the concerns in that situation is the patients who have left main disease, which constituted about $7 \%$ of the patients who eventually underwent CABG. Most of those were patients with left main disease. As you know, the stress test often will not detect left main disease because of balanced ischemia.

We have been relying less on nuclear stress imaging and more on computed tomographic angiography currently to study the coronary arteries. In those patients for whom we have a low index of suspicion for $\mathrm{CAD}$, we have mostly used cardiac computed tomographic angiography rather than even performing a stress test.

Dr Moon. That is 1 approach. We do perform stress tests for everybody, using an adenosine thallium nuclear test or dobutamine echocardiography. If those tests have negative findings, we do not perform catheterization. However, that is a different approach. 